\title{
Os fatores de riscos envolvidos no desenvolvimento da hipertensão infantil e suas consequências.
}

\author{
The risk factors involved in the development of infant hypertension and their \\ consequences.
}

\author{
Landri Antonio Neto Gomes ${ }^{1}$, Angela Falcai ${ }^{2}$
}

\begin{abstract}
Resumo: O objetivo dessa pesquisa consiste em avaliar, através de uma revisão sistemática, os fatores de riscos envolvidos no desenvolvimento da Hipertensão Arterial infantil. Caracterizar a epidemiologia da hipertensão arterial infantil. Discorrer sobre o diagnóstico e as indicações de tratamento para crianças portadoras de hipertensão arterial infantil. Trata-se de um artigo de revisão de literatura, descritiva, no qual foram analisados artigos já publicados sobre os fatores de riscos na HA infantil. Realizado busca nas bases de dados da PUBMED e SCIELO. Frequentemente a literatura elenca como fator de risco para HA o estilo de vida pouco saudável, como também outras características sócio demográfica são identificadas (econômica, fatores ambientais, etc.) e ponderam para elevação do IMC, desenvolvimento de diabetes mellitus e doenças cardiovasculares. Alguns estudos sugerem que a associação da hipertensão em crianças obesas, refere-se a ativação do sistema nervoso simpático, observando um desequilíbrio pelo deslocamento do mecanismo de controle da pressão arterial da diurese e a natriurese para maiores níveis de HA. Estudos ainda demonstram que a doença renovascular é uma das mais importantes causas da HA na infância, outras doenças como insuficiência cardíaca também sugerem relação embora poucos estudos sejam dirigidos a crianças. Observa-se que a prevalência desse agravo em crianças e adolescentes, vem causando muita preocupação na área da saúde. E constata-se que a alimentação saudável e a atividade física ainda são as maiores e melhores ferramentas para controle da HA.
\end{abstract}

Palavras chaves: Hipertensão. Infantil. Fatores de Risco. Tratamento.

\begin{abstract}
The objective of this research is to evaluate, through a systematic review, the risk factors involved in the development of Childhood Hypertension. This is a literature review, descriptive, unqualified article reviewed previously published on risk factors in childhood high blood. Carried out search in PUBMED and SCIELO databases. Often the literature as a risk model for unhealthy lifestyle, as well as other sociodemographic characteristics are identified (economic, environmental actors, etc.) and ponder for BMI elevation, development of diabetes mellitus and diseases cardiovascular diseases. Some studies suggest that an association of high blood pressure in obese children shows an imbalance due to the displacement of the diastolic blood pressure mechanism and natriuresis to higher levels of high blood pressure. Studies further demonstrate that renovascular disease are one of the most important causes of high blood in childhood, other diseases such as heart failure also suggest related sum studies are aimed at children. It is observed that it is a prevalence of aggravation in children and adolescents, it has been causing great concern in the health area. And it is found that healthy eating and physical activity are still bigger and better tools for high blood control.
\end{abstract}

Keywords: High blood pressure. Childhood. Risk Factors. Treatment.

\footnotetext{
1 Graduando do 11ํ do Curso de Medicina da Universidade Ceuma São Luís, Maranhão, Brasil.

2 Professora Doutora - Mestrado em Meio Ambiente - Universidade Ceuma. São Luís, Maranhão, Brasil.

Autor Correspondente:

Angela Falcai. Email: afalcai@gmail.com

Av. Deputado Luís Eduardo Magalhães, 1, Cond. Jardins Andaluzia, Torre Granada, Apto 204. Bairro

Calhau. Cep 65071-415. São Luís - Maranhão
} 


\section{Introdução}

A Hipertensão Arterial Sistêmica Infantil (HASi), é uma síndrome que apresenta algumas similaridades com a HAS no adulto. ${ }^{1}$ Entretanto, enquanto a HAS em sua grande maioria em adultos é de origem primária, a HASi é mais comum na forma secundária além de ser consideravelmente rara com uma prevalência média de até $5 \%$, mas a tempo é importante ressaltar sua notável prevalência na forma primária, de maneira assintomática sem alertar para a presença da doença. ${ }^{1}$

A Hipertensão Arterial Sistêmica (HAS), dentre as doenças cardiovasculares, é a mais prevalente e a que com frequência está associada a alterações metabólicas atingindo uma prevalência média de até $30 \%$ em adultos com mais de trinta anos, podendo gerar o aumento do risco para desenvolvimento das doenças circulatórias fatais e não fatais, insuficiência renal e entre outras. ${ }^{2}$

Em geral as doenças crônicas transmissíveis (DCT), classificam-se como a principal causa de morbimortalidade, representando em média $65 \%$ das mortes no mundo todo. ${ }^{3}$ Dentre elas, as doenças cardiovasculares de origem circulatória (hipertensão arterial, infarto, acidente vascular encefálico) destacam-se pela prevalência, sendo que aproximadamente um terço das mortes globais decorre por esse motivo. ${ }^{3}$

Dados da Organização Mundial da Saúde (OMS), revelam que aproximadamente 600 milhões de pessoas possuam HAS e que em média ocorram 7 milhões de mortes ao ano em função dessa enfermidade. ${ }^{1} \mathrm{Na}$ literatura os estudos demonstram que haverá um crescimento de $60 \%$ dos casos da doença até o ano de 2025. ${ }^{1}$

Assim, esses estudos corroboram com a ideia de que a HAS é um grande problema de saúde pública, despendendo um notável e acentuado aumento dos custos no Sistema Único de Saúde (SUS), afetando a economia mundial. $^{3}$

Via de regra a HASi é uma doença hereditária, crônicodegenerativa com graves lesões sistêmicas podendo condicionar lesões em órgãos alvo. Os estudos que visam avaliar a HASi denotam importância, ao passo que ilustram a magnitude da doença, visando estratificar os pacientes que necessitem de uma adequada intervenção. ${ }^{1}$

Assim o trabalho se justifica pela necessidade de uma intervenção galgada em prevenir 0 aparecimento de lesões em órgãoalvo e demais comorbidades a partir da identificação dos principais fatores de risco para a HASi.

Assim 0 objetivo desse trabalho consiste em realizar uma revisão sistemática sobre os fatores de riscos envolvidos no desenvolvimento da hipertensão arterial infantil.

\section{Estratégia de revisão}

Trata-se de um artigo de revisão de literatura sistemática, no qual foram analisados artigos já publicados sobre Hipertensão Arterial Infantil correlacionada com fatores de risco envolvidos e suas consequências. Realizado nas bases de dados da PUBMED e SCIELO, dos artigos publicados no período de 2003 a 2017. 
A estratégia de busca eletrônica teve os seguintes descritores "hipertensão arterial infantil", "fatores de risco da hipertensão infantil", "consequências da hipertensão infantil", e suas correspondentes em inglês "high blood pressure in childhood", "risk factors for childhood high blood pressure", "consequences of childhood hypertension". Foram incluídas publicações em língua portuguesa, inglesa e espanhola. Os artigos foram pré-selecionados a partir da leitura do resumo disponível.

Após a consulta às bases de dados, foram identificados estudos que apresentavam duplicidade entre as bases. Os artigos selecionados foram organizados, e analisados sistematicamente, depois foi elaborada uma planilha no programa Microsoft Office Excel®, contendo os descritores: Autoria, título, objetivo (Quadro 1).

\section{Revisão discutida}

Identificada como um dos precursores mais comuns da doença arterial coronariana, a HAS geralmente é assintomática e a prevenção é a maneira mais eficiente de lutar contra ela e evitar o alto custo social de seu tratamento e complicações. ${ }^{4}$

Entretanto é importante definir, a real necessidade da aferição dos níveis pressóricos e assim identificar os indíviduos com pressão arterial (PA) elevada, sendo um fator diagnóstico essecial. ${ }^{3}$

Em termos epidemiológicos constata-se que a prevalência mundial de HASi é de aproximadamente $2 \% \quad$ - $13 \%$, oscilando de acordo com a metodologia utilizada ${ }^{5}$. No Brasil, por exemplo, estima-se que a prevalência de HASi é de $4 \%{ }^{5}$.
Com isso, ainda afirma-se a necessidade de aferição da PA como medida empirica a partir dos três anos de idade, a PA tende a aumentar com o avançar da idade, e quando uma criança apresenta elevações no nível pressórico, remete a uma previsão de HAS na idade adulta, tendo seu fator iniciador ainda na infância. ${ }^{5,6}$

Via de regra, a PA deve ser validada e interpretada como resultado impactante das influências ambientais e de fatores patológicos sobre a expressão de vários genes que, por sua vez, expressam e regulam outros. ${ }^{5}$

A tempo verifica-se a expressão do gene da enzima conversora da angiotensina (ECA) e pela expressão do gene endotelial de Óxido Nítrico (NO). ${ }^{5}$

0 "4" Relatório sobre 0 Diagnóstico, Avaliação e Tratamento da Pressão Elevada em Crianças e Adolescentes", define critérios para caracterização de um diagnóstico de HASi, visto que uma única aferição adequada, não é fator determinante para diagnóstico ${ }^{7,8}$.

Dessa forma, tais crianças só podem ser consideradas hipertensas depois de que se realizem no mínimo três aferições consecutivas, e que essas sejam realizadas em situações e ambientes diferenciados, ao passo de que então se confirme 0 aumento da PA. ${ }^{9}$

$A$ avaliação dos níveis da PA na infância, leva em consideração critérios de ordem estatística, tendo como referencial tabelas que concentrem os valores normais da PA (Sistólica e Diastólica), em crianças que apresentam mesma idade, sexo e percentil de estatura ${ }^{7,8,9,10}$. 


\section{Quadro 1. Análise sistemática de publicações sobre Hipertensão Arterial Sistêmica Infantil.}

\begin{tabular}{|c|c|c|}
\hline $\begin{array}{l}\text { Autores (Ano) / } \\
\text { Base de dados }\end{array}$ & Título & Objetivos \\
\hline $\begin{array}{l}\text { WRITING } \\
\text { MEMBERS, Go AS, et al. } \\
(2014) / \text { PUBMED }\end{array}$ & $\begin{array}{l}\text { Heart Disease and Stroke } \\
\text { Statistics - } 2014 \text { Update } \\
\text { A Report From the American } \\
\text { Heart Association }\end{array}$ & $\begin{array}{l}\text { Atualização Estatística } \\
\text { Doenças Cardíacas }\end{array}$ \\
\hline $\begin{array}{l}\text { Prasad S, et al. (2017) / } \\
\text { PUBMED }\end{array}$ & $\begin{array}{l}\text { Elevated blood pressure and its } \\
\text { associated risk factors among } \\
\text { adolescents of a North Indian City } \\
\text { - A cross-sectional study }\end{array}$ & $\begin{array}{l}\text { Avaliar a prevalência de PA } \\
\text { elevada entre crianças e verificar } \\
\text { os fatores de risco associados. } \\
\text { Avaliar o conhecimento das } \\
\text { crianças e adolescente sobre a PA } \\
\text { e seus fatores modificáveis. }\end{array}$ \\
\hline $\begin{array}{l}\text { Malta DC, et al (2017) / } \\
\text { SCIELO. }\end{array}$ & $\begin{array}{l}\text { Prevalence of and factors } \\
\text { associated with self-reported high } \\
\text { blood pressure in Brazilian adults }\end{array}$ & $\begin{array}{l}\text { Analisar fatores associados à } \\
\text { hipertensão arterial auto-relatada } \\
\text { entre adultos em capitais } \\
\text { brasileiros. }\end{array}$ \\
\hline
\end{tabular}

Ferreira HS, et al. (2015) / High Blood Pressure among PUBMED Schools in Maceió, Brazil.
Avaliar a prevalência de hipertensão arterial e fatores associados (socioeconômicos e antropométrica) crianças matriculadas em escolas públicas e privadas de Maceió, Brasil.
Rodrigues AN, et al. (2013) / PUBMED

Raj M. (2011) / PUBMED

Bloetzer C, et al. (2015) /
PUBMED
Cardiovascular risk factor investigation: a pediatric issue.
Correlacionar fatores de risco cardiovascular na infância e adolescência com a ocorrência de doença cardiovascular.

Essential hypertension in Apresentar pesquisas recentes na adolescents and children: Recent etiopatogenia básica da advances in causative hipertensão essencial em crianças mechanisms. e adolescentes.

Performance of targeted screening for the identification of hypertension in children.

Avaliar o desempenho da história combinada de hipertensão arterial e sobrepeso / obesidade para identificar crianças com hipertensão.

Estudar a prevalência de hipertensão arterial (HA) e avaliar os fatores de risco e as complicações associadas à doença em crianças e

adolescentes.

Crispim PAA, et al. (2014) / Fatores de Risco Associados aos PUBMED

Níveis Pressóricos Elevados em Crianças de Dois a Cinco Anos
Avaliar a prevalência de PA alta e fatores associados em crianças.
Kupferman JC, et al. (2016) Stroke and Hypertension in /PUBMED

Children and Adolescents.
Salgado CM, Carvalhaes JTA. (2003) / SCIELO
Hipertensão arterial na infância.
Resumir a literatura que pode vincular a pressão arterial elevada ao desenvolvimento de AVC isquêmico e hemorrágico na infância

Realizar uma revisão crítica da literatura atual, enfocando aspectos práticos e relevantes para o diagnóstico e tratamento ambulatorial da criança com hipertensão arterial. 
Via de regra, crianças que apresentam percentil abaixo de 90 caracterizam-se como portadores de PA normal, as que apresentam percentil entre 90 e 95, são categorizadas como quadro de préhipertensão e aquelas com percentil acima de 95 são as que apresentam a HASi já instalada. 5,6

Alguns critérios também foram definidos, como o de que toda criança maior de três anos deve ter sua PA avaliada rotineiramente em consultas médicas e aquelas que apresentam menos de três anos devem ter sua PA aferida quando há suspeição de hipertensão secundária mediante a existência de outra comorbidade. ${ }^{5}$

Além de é claro considerar os padrões internacionais de aferição de PA, que exige principalmente ambiente silencioso e arejado, bexiga totalmente esvaziada, repouso por no mínimo 5 minutos, paciente sentado com costas apoiadas, pernas sem cruzar e braços esticados na altura do coração. ${ }^{5}$

A escolha do manguito deve ser o pediátrico, visto que o mesmo é o indicado por possuir tamanhos relativamente adequados a circunferência do braço da criança, além da necessidade de ser calibrado em intervalos de tempo específicos, para manter a sua aferição fidedigna. ${ }^{5,10}$

- Vários fatores conhecidos relacionados à HAS em adultos, como sexo, idade, história familiar e presença de aumento do peso corporal ou obesidade são também observados na HASi. ${ }^{5,10,11,12}$. A alta PA contribui para 0 desenvolvimento de complicações cardiovasculares. $\quad 5,10 \quad$ Sua associação com múltiplos fatores de risco tem um efeito multiplicador sobre 0 risco de eventos cardiovasculares. ${ }^{5,10,11,12}$

Frequentemente a literatura elenca como fator de risco para HAS o estilo de vida pouco saudável, chamando atenção para os hábitos alimentares, que acabam sendo muitas vezes inadequados associado a falta de atividade física. $5,10,11,12$

Assim, outras características como baixo nível socioeconômico, fatores genéticos, fatores ambientais e fatores antropométricos, ponderam para elevação do IMC, desenvolvimento de diabetes mellitus e doenças cardiovasculares. ${ }^{11,12}$

Estima-se que $30 \%$ das crianças e adolescentes com sobrepeso/obesidade tenham hipertensão. 5,13 Assim, a presença de sobrepeso/obesidade parece ser um dos fatores mais importantes relacionados à hipertensão em crianças e adolescentes em todo o mundo. Vários estudos mostraram que a presença de sobrepeso/obesidade está positivamente correlacionada com a ocorrência de pré-hipertensão em crianças, e esta combinação aumenta o risco de desenvolver HAS de adultos. ${ }^{5,13}$

Alguns desses estudos ainda sugerem que a associação da hipertensão em crianças obesas, refere-se a ativação do sistema nervoso simpático, observando um desequilíbrio pelo deslocamento do mecanismo de controle da pressão arterial da diurese e a natriurese para maiores níveis de HAS. ${ }^{1,14}$

Certas condições estão fortemente associadas à HAS, muitas vezes pouco encontradas na infância, mas não exclusas dessa época de vida, a saber, fumar e o uso de contraceptivos, álcool, 
cocaína, anfetaminas, esteróides anabolizantes, fenilpropanolamina e pseudoefedrina

(descongestionantes nasais), com isso no geral podemos classificar como fatores preponderantemente adaptaveis e adequaveis. ${ }^{1}$

Via de regra, os principais determinantes da PA elevada destacado na literatura são as variáveis que incluem o fator dietético, o sobrepeso/obesidade, o sedentarismo e presença da herança genética como fator predisponte. $^{5,10,11,12}$

Com isso é possível ressaltar as principais consequências encontradas por essas variáveis a começar pelo fator dietético, destacando a ingesta de sódio e potássio. ${ }^{11}$

A forte relação entre a ingesta de sódio e HAS se destaca por um estudo que avaliou a redução da PAS (em aproximadamente 5 $\mathrm{mmHg}$ ) e PAD (em 2,5 mmHg) em pacientes hipertensos, após a restrição de sódio na dieta. ${ }^{11} \mathrm{~A}$ tempo o mesmo estudo avaliou a redução também em pacientes normotensos sendo PAS (em aproximadamente $2 \mathrm{mmHg}$ ) e PAD ( em aproximadamente $1 \mathrm{mmHg}$ ), 0 que infere dizer que embora pequena verifica-se uma pequena correção pressórica. ${ }^{11}$

Em outro estudo, foi contrastado que em crianças, objeto desse estudo, a restrição de sódio é mais efetiva nas que apresentam história familiar de HAS e também aquelas crianças que se apresentam com sobrepeso/obesidade diferindo do restante da população infantil avaliada. ${ }^{10}$

Entretanto, vale ressaltar que somente a alta ingestão de sódio não é determinante para que se verifique a $\mathrm{HA}$, outro determinante $\mathrm{e}$ principal consequência da ingesta de sódio é verificada pela sensibilidade de cada pessoa ao sódio. ${ }^{11} \quad$ Algumas pessoas apresentam menor sensibilidade ao sódio e com isso excretam menor quantidade desse eletetrólito, provocando um aumento na PA, visto que o aumento da ingesta de sódio resulta na diminuição da atividade do Sistema ReninaAngiotensina-Aldosterona (SRAA) e consequente aumento na sintese e liberação do Peptídeo Natriurético Atrial. 1

Por outro lado no que se refere a ingestão de potássio, estudos contrastam que a sua suplementação tem papel importante na prevenção da HA, visto que através de uma dieta com suplementação de potássio esta associada a uma redução significantes da PAS e PAD. ${ }^{10,11,12}$

O mesmo mecanismo observado como processo de sensibilização pelo sódio ocorre também com o potássio, dessa maneira em outro estudo foi demonstrado que a determinação da PA em crianças e adolescentes apresenta maior importância quando associado ingesta e restrição de sódio e potássio, respectivamente ${ }^{11,12}$. Do que ao se observar a ingesta de sódio isoladamente ${ }^{11,12}$.

Já no que diz respeito a variável, sobrepeso/obesidade, denota sua principal consequência a predisposição e associação com doenças silenciosas e muitas vezes subdiagnosticas na infância como a diabetes melittus II, a dislipidemia e a síndrome da resistência a insulina $^{10,12}$.

Os mecaniscos destacados para a presença de HA nessas crianças são classificados como complexos, sendo que as principais 
alterações

indentificadas

fisiopatológicas

hiperinsulinemia compensatória, ativação do sistema nervoso simpático e SRAA e também as de cunho hemodinâmico ${ }^{11,12}$.

Outra variável ainda de forte vertente é o sedentarismo, com o advento de tecnologias interativas as crianças acabam se tornando mais sedentárias, por acabarem excluindo de suas atividades lúdicas brincadeiras que envolvam gasto energético a partir de atividades aeróbicas como corrida, futebol e andar de bicicleta ${ }^{1,10,11,12}$. Dessa maneira estudos demonstraram forte relação para que essas crianças criem maior predisposição para 0 desenvolvimento de sobrepeso/obesidade, além do aumento da gordura visceral ${ }^{1,10,11,12}$.

Por fim a ultima variável fortemente descrita na literatura é o herança genética, vários estudos em crianças e adolescentes mostraram que a avaliação da PA em filhos de pais hipertensos em relação a filhos de pais normotensos, são maiores. ${ }^{10,11,12}$

Outra condição genética ilustrada em um estudo foi de que, pessoas expostas a estressores psicológicos apresentam maior predisposição a ter HA. ${ }^{12}$ Sendo que 0 fator genético influencia a resposta ao estresse, visto que os filhos de pais hipertensos tendem a apresentar o aumento da PA como resposta a fatores de estresse ${ }^{12}$.

O gráfico 1, apresenta a Frequência Relativa de uma amostra e a associação da PA elevada dessa amostra em um estudo, em que foi observado que a predisposição da HASi é maior em crianças com menos de 2 anos de idade, crianças filhas de mães que apresentam escolaridade menor que 9 anos em tempo de estudos e aquelas crianaças classifficadas como sobrepeso/obesas pelo IMC, contracenando a disposição ja citada averiguada em outros estudos. ${ }^{12} \mathrm{Em}$ especial no estudo acima essas foram as variávies que demontraram maior grau de significância para um estudo de a: de $5 \% .^{12}$

Mas ainda vale citar a presença de fatores patológicos, como a hipertensão renovascular (HRV), que é considerada uma das importantes causas de HASi, um trabalho descreve que sua prevalência é comum em média de $10 \%$ dos casos, além de caracterizar que a detecção e o tratamento adequado da HRV, como principal causa de HASi, são de extrema importância a fim de prevenir doenças como a nefropatia isquêmica, doença renal crônica (DRC) e dano de órgão alvo ${ }^{15}$.

A insuficiência cardíaca também sugere relação com a HAS, embora poucos sejam os estudos dirigidos a crianças, o que chama a atenção para necessidade de estudos que busquem um contraste a esse cenário. ${ }^{15}$

Como já introduzido, a prevalência da hipertensão secundária é maior na HASi, em um estudo realizado foi possível inferir que crianças menores de 6 anos de idade estão mais propensas a desenvolverem hipertensão secundária, e em geral elas estão menos propensas a apresentarem peso ou IMC > que o percentil 95 quando comparadas com crianças mais velhas. ${ }^{16}$

Outro dado relevante se expressa na taxa de filtração glomerular, que se mostrou menor em crianças menores que 6 anos de idade, entretanto 0 colesterol destaca-se por apresentar menores concentrações nesse grupo, o que 
infere dizer que essas crianças apresentaram menor predisposição

a serem obesas ${ }^{16}$.

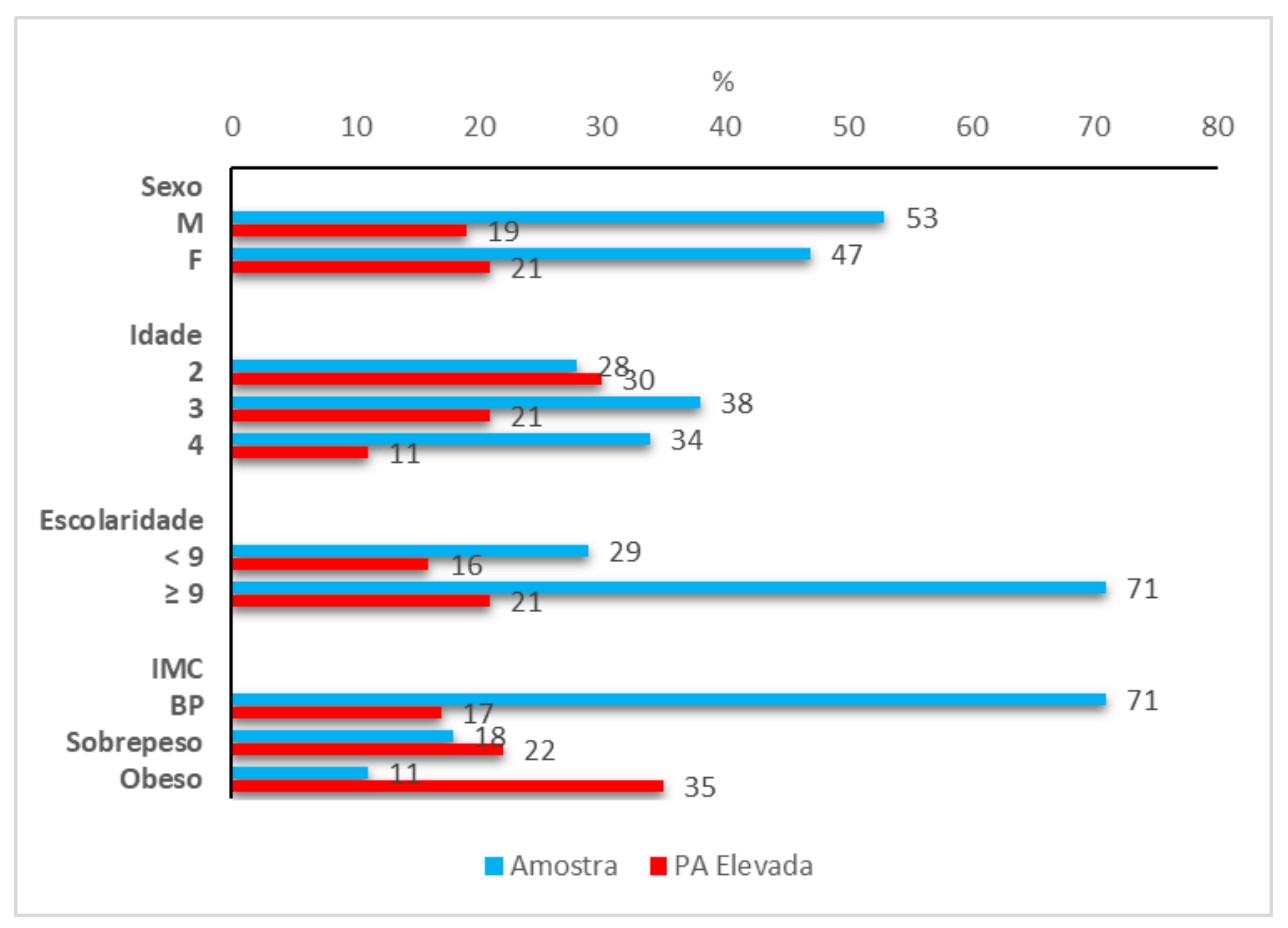

Gráfico 1. Frequência relativa de uma amostra e associção da PA a essa amostra de um estudo (Adaptado)

Portanto, as mudanças no estilo de vida, como controle de peso, redução na ingestão de sódio e exercício, são fundamentais para a prevenção da hipertensão., ${ }^{1,5}$ Embora os níveis de pressão limiar ainda não estejam bem definidos, a PA provavelmente afeta os órgãos.alvo em crianças, como em adultos. ${ }^{1,5} \mathrm{~A}$ intervenção dietética, a manutenção de um peso ideal e o envolvimento em atividades físicas regulares poderia ser encorajada nesta etapa como um método de prevenção primária. ${ }^{1,5} \mathrm{Em}$ um estudo sobre a rigidez dos vasos arteriais grandes, que é atribuído ao envelhecimento, o único fator estudado que poderia explicar 0 grupo com vasos que não mostravam sinais de idade era 0 menor nível de PA apresentado ${ }^{1,5}$.

A hipertensão é um problema importante de saúde pública, que representa 7,6 milhões de mortes em todo o mundo anualmente, $80 \%$ dos quais ocorrem em países em desenvolvimento como o Brasil. ${ }^{1}$ Cerca de $54 \%$ e $47 \%$ dos casos de AVC e infarto do miocárdio, respectivamente, em todo o mundo estão associados à hipertensão ${ }^{1}$.

Com isso observa.se que 0 tratamento não farmacológico via de regra é o ideal e sempre deverá ser priorizado e, por ser de primeira escolha ainda, mostra.se muito eficaz no controle da PA. ${ }^{10}$ Entretanto, algumas vezes faz.se necessária a utilização e inclusão 
de medicamentos anti-hipertensivos no combate da HAS ${ }^{10}$.

Nesse caso, várias classes terapêuticas podem ser utilizadas a fim de controlar a PA, a saber, são utilizados diuréticos, betabloqueadores, inibidores da enzima conversora da angiotensina (IECAs), antagonistas do cálcio, bloqueadores dos receptores da angiotensina II (BRA II) e alfa bloqueador, entre outros ${ }^{17,18,19}$.

O primeiro passo que a equipe multidisciplinar deve ter em mente é o de iniciar o tratamento com apenas uma droga na dose adequada, 0 que demonstra resultados extremamente satisfatórios no controle da $\mathrm{PA}^{20,21}$.

Caso seja observado pela equipe que não esteja ocorrendo 0 controle desejado, acrescenta.se uma segunda droga, visto que na literatura pouquíssimos estudos referem à utilização de mais de um medicamento para o controle de PA nas crianças e adolescentes, o que demonstra pouca necessidade $e$ que a monoterapia medicamentosa é eficaz ${ }^{19}$.

Poucos são os estudos em longo prazo sobre o tratamento farmacológico da $\mathrm{HASi}^{10}$ Mas, algumas considerações podem ser estabelecidas, com base nos medicamentos que possam ser utilizados ${ }^{10}$.

É importante definir e separar as medicações anti-hipertensivas em dois grandes grupos, as que irão ser empregadas em emergências hipertensivas e as que serão empregadas no tratamento crônico $^{19,20,21}$.

Quanto às emergências hipertensivas podemos citar 0 nifedipino como bloqueador dos canais de cálcio, que é a primeira droga a ser largamente utilizada em crianças, principalmente pela sua ação rápida, é um agente diiidropiridínico que apresenta potencial para diminuição da força de contração cardíaca, os efeitos adversos são predominantemente no inicio do tratamento, caracterizados em geral como leves e transitórios, que incluem taquicardia, cefaleia, rubor facial e vertigem. ${ }^{20,21}$

Nesse grupo também incluímos Nicardipina que é outro bloqueador dos canais de cálcio, que apresenta um efeito adequado na crise hipertensiva, sendo utilizado para aliviar e controlar a angina $^{19,20,21}$.

O Nitroprussiato de Sódio é um vasodilatador direto, utilizado nas crises hipertensivas por seu efeito imediato, com o intuito de reduzir agudamente a pré e/ou a pós.carga, se for administrado por mais de $72 \mathrm{~h}$, existe o risco de acúmulo de um metabólito denominado tiocinato, sendo assim faz se necessário o controle dos níveis séricos desse metabólito ${ }^{20,21}$.

O Labetalol é um bloqueador adrenérgico simultâneo dos receptores alfa $e$ beta em proporções fisiologicamente equilibradas, por esse efeito sinérgico produz vasodilatação, diminuição da resistência vascular e assim contribui para a diminuição da PA, além da proteção miocárdica das respostas reflexas indesejadas pelo bloqueio do receptor beta ${ }^{20,21}$.

Por fim o esmolol é betabloqueador injetável que se caracteriza por apresentar efeitos adversos como broncos espasmos, náuseas e vômitos além de reação local ${ }^{20,21}$.

Quanto ao uso das medicações para o tratamento crônico podemos citar IECAs por apresentarem efeitos favoráveis nas funções miocárdicas e renais, até o 
ano de 2000 não havia sido aprovado para tratamento em crianças hipertensas sendo que o primeiro aprovado foi o enalapril, que é bem tolerado, apesar de poder provocar tosse seca, hipotensão postural, cefaleia, tontura e fadiga. $^{20,21}$ A sua vantagem em relação ao captopril é o numero de administrações diárias e a sua biodisponibilidade não ser influenciada pelos alimentos. ${ }^{20,21}$

Os antagonistas dos canais de cálcio como o anlodipina e isradipina são bem tolerados, apropriados aos pacientes com insuficiência renal crônica, apresentam como reações adversas palpitações, hipotensão, taquicardia. $19,20,21$ O anlodipina é um diidropiridínico mais recente e de longa ação que apresenta como vantagem em relação aos mais antigos, um efeito mais prolongado, suave e não associado a taquicardia reflexa nem ao aumento de catecolaminas. ${ }^{19,20,21}$

Entre os diuréticos, o mais utilizado é a hidroclorotiazida, devido ao seu custo/beneficio, sua utilização não deve ser em altas doses por estar associado ao aumento de triglicerídeos, da glicemia, do ácido úrico, deve.se também monitorizar a diminuição de potássio ${ }^{20,21}$. Podemos citar também o uso de furosemida, bumetanida e espironolactona, que também devem ser assistidos por apresentarem os mesmo efeitos em grandes concentrações e com atenção maior a espironolactona que é um antagonista da aldosterona. ${ }^{20,21}$

Os betabloqueadores como o propranolol não devem ser utilizados em asmáticos. ${ }^{20,21}$ Nesse grupo de pacientes, o uso de beta adrenérgico e corticosteroides sistêmicos devem ser desencorajados,

dando.se preferência às preparações inalantes ${ }^{20,21}$.

\section{Conclusão}

De acordo com os estudos e revisão, destaca.se o reconhecer precocemente da pressão arterial anormal na infância e a intervenção (prevenção, investigação e tratamento) adequada é necessária para diminuir a morbidade/ mortalidade além de prevenir problemas cardiovasculares e renais futuros.

Ainda é possível fundamentar que as crianças hipertensas com idade menor que 6 anos são mais propensas a apresentarem HAS secundária além de apresentarem maior PA diastólica e menor TFG, estando menos propensas a obesidade devido ao baixo colesterol.

Por isso deve.se levar em consideração as orientações do "Relatório sobre o Diagnóstico, Avaliação e Tratamento da Pressão Elevada em Crianças e Adolescentes", que dispõe de condutas e normatizações para 0 adequado diagnostico da HASi.

Dessa maneira, constata.se que o acompanhamento de uma equipe multidisciplinar tem um principio e importância considerável, pois dessa forma quando a equipe aborda pacientes que apresentam HASi, devem ter como objetivo cuidar e combater as necessidades imediatas da saúde. No entanto, não podem excluir de seus objetivos - princípio da prevenção e monitorização das doenças futuras, que podem ter a HASi como precursora.

A HASi insere.se nesse contexto, visto que o tratamento não 
farmacológico é eficaz na grande maioria das vezes, embora um pequeno número de pacientes necessite de apenas um fármaco e outro número ainda menor de pacientes, precisará usar mais de uma droga.

Por fim, conclui.se que nessa fase da vida, é de fundamental importância e interesse da área médica a prevenção de doenças degenerativas que se iniciam na infância e que podem acometer 0 indivíduo na idade adulta, tirando.lhe a tranquilidade de um envelhecimento saudável que é esperado como perspectiva de vida.

\section{Referências}

1. Prasad S, Masood J, Srivastava AK, Mishra P. Elevated Blood Pressure and Its Associated Risk Factors among Adolescents of a North Indian City. A Cross.sectional Study. Indian Journal of Community Medicine: Official Publication of Indian Association of Preventive \& Social Medicine. 2017;42(3): $155.158 . \quad$ doi:10.4103/ ijcm.IJCM_106_16.

2. Go AS, Mozaffarian D, Roger VL, Benjamin EJ, Berry JD, Blaha MJ, et al. Heart disease and stroke statistics - 2014 update: A report from the American heart association. Circulation. 2014;129:e28-92.

3. Malta, DC, et al. Prevalência e fatores associados com hipertensão arterial autorreferida em adultos brasileiros. Rev. Saúde Pública, São Paulo , v. 51, supl. 1, 11s, 2017. Available from <http://www.scielo.br/scielo.php?scri pt=sci_arttext\&pid=S0034.89102017 $000200313 \&$ Ing $=$ en\&nrm=iso $>$. access on 07 Oct. 2017. Epub June 01, 2017. http://dx.doi.org/10.1590/ s1518.8787.2017051000006.
4. Ferreira HS, Lúcio GMA, Assunção $\mathrm{ML}$, et al. High Blood Pressure among Students in Public and Private Schools in Maceió, Brazil. Kokubo Y, ed. PLoS One. 2015;10(11:e0142982. doi:10.1371/journal.pone.0142982.

5. Rodrigues AN, Abreu GR, Resende RS, Goncalves WL, Gouvea SA. Cardiovascular risk factor investigation: a pediatric issue. International Journal of General Medicine. 2013;6:57.66. doi:10.2147/IJGM.S41480.

6. Raj M. Essential hypertension in adolescents and children: recent advances in causative mechanisms. Indian $J$ Endocrinol Metab. 2011;15(Suppl 4):S367-S373.

7. Bloetzer C, Bovet P, Paccaud F, Burnier M, Chiolero A. Performance of targeted screening for the identification of hypertension in children. Blood Press. 2017 Apr. 26(2):87.93.

8. Kupferman JC, Zafeiriou DI, Lande MB, Kirkham FJ, Pavlakis SG. Stroke and hypertension in children and adolescents. J Child Neurol. 2017 Mar. 32(4): 408.17.

9. National High Blood Pressure Education Program Working Group on High Blood Pressure in Children and Adolescents. The fourth report on the diagnosis, evaluation, and treatment of high blood pressure in children and adolescents. Pediatrics. 2004 Aug. 114(2 Suppl 4th Report):555.76.

10. Salgado, Cláudia Maria; Carvalhaes, João Thomaz de Abreu. Hipertensão arterial na infância. J. Pediatr. (Rio J.), Porto Alegre, v. 79, supl. 1, p. S115.S124, June 2003. Available from $<$ http://www.scielo.br/scielo.php?scri pt=sci_arttext\&pid=S0021.75572003 $000700013 \& \operatorname{lng}=$ en\&nrm $=$ iso $>$. access on 22 jul. 2017. http://dx.doi.org/10.1590/S0021.755 72003000700013. 
11. N FS, Cândido APC. Prevalência e fatores de risco associados à hipertensão arterial em crianças e adolescentes: uma revisão de literatura. HU Revista, Juiz de Fora, v. 39, n. 1 e 2, p. xx.xx, jan./jun. 2013.

12. Crispim PAA, et al. Fatores de Risco Associados aos Níveis Pressóricos Elevados em Crianças de Dois a Cinco Anos. Arq. Bras. Cardiol., São Paulo, v. 102, n. 1, p. 39.46, Jan. 2014.

13. Srinivasan SR, Myers L, Berenson GS. Changes in metabolic syndrome variables since childhood in prehypertensive and hypertensive subjects: the Bogalusa Heart Study. Hypertension. 2006;48:33-39.

14. Batista MS, Mill JG, Pereira TSS, Fernandes CDR, Molina M del CB. Factors associated with arterial stiffness in children aged 9.10 years. Revista de Saúde Pública. 2015;49:23. doi:10.1590/S0034.8910.201504900 5425.

15. Chung $H$, Lee $J, H$, Park $E$, Hyun $H$, Ahn $Y, H$, Jae H, J, Kim G, B, Ha I, $S$, Cheong $H$, IKang $H, G$, Long.Term Outcomes of Pediatric Renovascular Hypertension. Kidney Blood Press Res 2017;42:617.627.

16. Flynn J, Zhang Y, Solar.Yohay S, Shi V. Clinical and Demographic Characteristics of Children With Hypertension. Hypertension. 2012;60:1047.1054, originally published September 12, 2012. https://doi.org/10.1161/HYPERTENS IONAHA.112.197525.

17. Shapiro DJ, Hersh AL, Cabana MD, Sutherland SM, Patel Al. Hypertension screening during ambulatory pediatric visits in the United States, 2000.2009. Pediatrics. 2012 Oct. 130(4: 604.10.

18. Brasil (Ministério da Saúde) . Coordenação de doenças cardiovasculares: Doenças cardiovasculares no Brasil. Sistema Único de Saúde, Brasília, 2010.
19. Kuschnir MCC, Ribeiro MG. Hipertensão arterial na adolescência: abordagem e tratamento. Adolesc Saude. 2006; 3(3: 7.10.

20. Bulário Explicativo / organizado por Rosana Cristina Spezia Ferreira. ISBN: 978.85.339.2852.7 - São Paulo: Rideel, 2013.

21. Pires EMSG. Hipertensão arterial em crianças e adolescentes. Diretrizes para o diagnóstico e tratamento. Sociedade Beneficente Israelita Brasileira. Diretriz Assistencial. DI.ASS.6.2. 2013. 